\title{
Syndromes with chronic non-bacterial osteomyelitis in the spine
}

\author{
Łukasz Kubaszewski ${ }^{1}$, Piotr Wojdasiewicz ${ }^{1,2,3}$, Marcin Rożek ${ }^{4}$, Iwona E. Słowińska², \\ Katarzyna Romanowska-Próchnicka ${ }^{3,5}$, Radosław Słowiński ${ }^{1}$, Łukasz A. Poniatowski ${ }^{3}$, Robert Gasik ${ }^{1,2}$ \\ ${ }^{1}$ Department of Neuroorthopaedics and Neurology, National Institute of Geriatrics, Rheumatology and Rehabilitation, Warsaw, Poland \\ 2Department of Rheumaorthopaedics, National Institute of Geriatrics, Rheumatology and Rehabilitation, Warsaw, Poland \\ ${ }^{3}$ Department of General and Experimental Pathology with Centre for Preclinical Research and Technology (CePT), Second Faculty \\ of Medicine, Medical University of Warsaw, Warsaw, Poland \\ ${ }^{4}$ Department of Orthopaedics and Traumatology, Hospital in Śrem, Śrem, Poland \\ ${ }^{5}$ Department of Connective Tissue Diseases, National Institute of Geriatrics, Rheumatology and Rehabilitation, Warsaw, Poland
}

\begin{abstract}
Chronic non-bacterial osteomyelitis (CNO) has been known for over of 40 years. It is an underrecognized entity due to the low number of described cases and poor propagation awareness of the problem. Chronic non-bacterial osteomyelitis is usually confused with infectious spondylodiscitis or malignant lesions, both primary and metastatic. Failing to consider CNO as one of possible lesions of the spine among an array of differential diagnoses may lead to a prolonged ineffective treatment increasing treatment-related morbidity. In this paper the authors describe these two syndromes, with a possible autoimmune background - chronic recurrent multifocal osteomyelitis (CRMO) and SAPHO syndrome - that include CNO being among the manifestations. The authors present the spinal symptomatology of CNO for both syndromes published so far to help spine clinicians organize the information for better usage in everyday clinical practice.
\end{abstract}

Key words: osteomyelitis, SAPHO syndrome, CRMO, non-bacterial inflammation.

\section{Introduction}

Chronic non-bacterial osteomyelitis (CNO) occurs most often as a part of two syndromes with putative autoimmune background. Although the syndromes have been known for over 40 years, due to the limited number of cases presented in the literature and differentiated symptomatology it is not uncommon that patients are misdiagnosed, diagnosed late and receive improper treatment. Most authors present the disease as characterized by an insidious onset, though with early symptoms in the spine resembling osteomyelitis or malignant lesions [1].

Diagnosis is based mainly on the clinical picture: localized pain and moderate systemic inflammation symptoms. In biopsy, CNO may be suspected if there are non-specific inflammatory changes accompanied by dermal manifestations together with the exclusion of other possible diseases. In some cases improper diagno- sis caused a prolonged ineffective antibiotic therapy or unnecessary surgical interventions [2]. Available literature referring to this particular topic is not consistent in terms of nomenclature and often presents poor practical scope for spine clinicians. Therefore, the aim of this paper is to present, based on the literature, concise and orderly information referring to the syndromes where one of the clinical manifestations is CNO in the spine.

\section{Nomenclature and classification system problems: an overview of the most commonly used terms for non-bacterial osteomyelitis}

In the literature three terms are mainly used: chronic recurrent multifocal osteomyelitis (CRMO), SAPHO syndrome and CNO. Historically, at first the term chronic

Address for correspondence:

Iwona E. Słowińska, Department of Rheumaorthopaedics, National Institute of Geriatrics, Rheumatology and Rehabilitation, Spartańska 1, 02-637 Warsaw, Poland, e-mail: i.s@onet.eu

Submitted: 31.10.2015; Accepted: 8.01.2016 
recurrent multifocal osteomyelitis was coined, which is a rare non-pyogenic inflammatory bone condition with unclear etiology observed in children [3].

The syndrome, with the main manifestation of osteomyelitis is accompanied by synovitis, acne, pustulosis - especially palmoplantar (PPP) and hyperostosis (primarily attributed to the clavicle with commonly occurring symmetrical involvement) [4]. The presentations of the syndrome may vary in: different time of onset of osseous and non-osseous symptoms where time span may be up to several years, monofocal or multifocal bone involvement, and in some cases the recurrence may even be unnoticed. Therefore, a more relevant term was presented: CNO or sterile bone inflammation.

Based on the literature and according to Ferguson et al. [5], the terms CRMO and CNO mainly refer to the younger population and are often used interchangeably in pediatric literature. According to Hofmann et al.: "CNO covers a wide clinical spectrum from non- or oligo-symptomatic monofocal bony lesions to the most severe CRMO form" [6]. The SAPHO syndrome (synovitis, acne, pustulosis, hyperostosis, osteitis) is basically the same syndrome as CRMO with more regular and pronounced skin symptoms $[7,8]$. The syndrome was named by Chamot et al. in 1987 [9].

In the literature the term is used mainly in older population. In some cases it is classified as a subtype of spondyloarthropathy due to sacroiliitis in some patients, as well as human leukocyte antigen B27 (HLA-B27) or human leukocyte antigen B8 (HLA-B8) were confirmed in limited cases [1, 7, 10-12]. Patients presenting SAPHO syndrome are usually adults in their 50-ties and 60-ties, or in some cases adolescents or children are included $[1,2,7,13]$. There is no agreement whether those syndromes are the same disease with different clinical symptoms. Some authors considered them as the same entity in different age groups [14-17]. In our opinion, it is best to use the term CNO as a specific lesion being the part of both CRMO and SAPHO syndrome. In the article we separately review the literature presenting both CRMO and SAPHO syndrome with special focus on the spinal presentations of CNO.

\section{Chronic recurrent multifocal osteomyelitis}

Chronic recurrent multifocal osteomyelitis was described primarily in children and adolescents [18]. Mean age at CRMO onset is approximately 10 years, with a range of 4-14 years, mean disease duration is approximately 5 years, and the mean number of flares per patient was approximately 6 [19]. Most studies reported a predominance in female patients, which is as high as up to $85 \%$ in Scully et al. group, or female to male ratio of $5: 1$, but the majority of literature presentations contain case reports [19-21].
The etiology of most CRMO cases is not known. The episodes of systemic inflammation occur due to immune dysregulation without autoantibodies, pathogens or antigen-specific T cells [18]. The infantile onset of CRMO is connected with a genetic mutation in Majeed syndrome and the deficiency of interleukin 1 receptor antagonist (DIRA) [5].

CRMO is characterized by the non-specific onset of pain with swelling and tenderness over the affected bone or joint, worsening at night. In long bones the metaphyseal regions are affected. The most common locations of osteomyelitis are: distal femur, proximal tibia, distal tibia, and distal fibula $-34 \%$, followed by the clavicle $-24 \%$ (historically first described and considered as classic for the syndrome), chest wall - 13\%, vertebral bodies from 2 to $8 \%$, mandible, pelvis - 14\%, shoulder girdle, and small bones of the hands [14, 20, 22, 23]. The lesions may occur in any bone [18]. The syndrome may be limited to a solitary bone lesion with only long bone or mandible presentation [24, 25]. Symmetric involvement is often indicated as the characteristic trait [6]. Associated skin manifestations are: PPP, generalized pustulosis, psoriasis vulgaris and acne [5]. Laboratory tests, such as white blood cell (WBC) count, erythrocyte sedimentation rate (ESR) and tumor necrosis factor $\alpha$ (TNF- $\alpha$ ) levels may be mildly elevated [5].

Differential diagnosis should include acute haematogenous osteomyelitis (CRMO represents 2-5\% of all osteomyelitis cases in children and adolescents), neoplasms, especially Ewing sarcoma, eosinophilic granuloma, osteoblastoma, osteoid osteoma as well as Langerhans cell histiocytosis or insufficiency fractures [11, 19, 26]. The possibility of mimicry working both ways also needs to be remembered [27]. The diagnosis is based on clinical criteria [6].

\section{Natural history of chronic recurrent multifocal osteomyelitis}

The natural history of the disease is unpredictable with varying severities and time courses, oscillating between acute exacerbations and spontaneous remission $[3,28]$. As stated before, owing to the lack of a diagnostic test, CRMO is diagnosed via exclusion [29-31]. Long-term clinical outcomes for children with CRMO appear to be generally good, with most subjects having no evidence of disease activity or sequelae. However, in a number of subjects the disease is persistent and the risk of physical and psychological complications is considerable. Further research is required to identify patients at risk for persistent disease, and to determine therapies that may prevent morbidity [19]. In spite of the self-limiting character of the syndrome, in some cases it may have a prolonged course and result in significant 
morbidity related to bone deformation but also to unnecessary diagnostic procedures (biopsy) and treatment (prolonged antibiotic therapy, surgical debridement) [29, 31]. In most children the onset of CRMO occurs at a later age and may be concomitant with psoriasis or inflammatory bowel disease (IBD) [5]. Permanent bone deformity may also occur, particularly when vertebral bodies are involved, often leading to more aggressive treatment. In most cases CRMO is a disorder that resolves after many years, most commonly without any permanent sequelae.

\section{Chronic recurrent multifocal osteomyelitis - associated inflammatory syndromes}

Systemic inflammatory syndromes which co-exist with CRMO are: Crohn's disease, ulcerative colitis (UC), celiac disease [5]. Other, less common syndromes include Sweet's syndrome, dyserythropoietic anemia, pyoderma gangrenosum, sclerosing cholangitis, inflammatory arthritis, sacroiliac joint involvement, Still's disease, Takayasu's disease, antineutrophil cytoplasmic antibody-positive (ANCA-positive) vasculitis, Ollier disease (multiple enchondromatosis), parenchymal lung disease, dermatomyositis, and tumoral calcinosis [5].

\section{Blood cultures and biopsy of the bone analysis in chronic recurrent multifocal osteomyelitis}

Cultures of blood bone biopsy are in general negative, even with polymerase chain reaction (PCR) analysis [5]. Histopathological findings are non-specific and demonstrate lymphocyte infiltration with neutrophilic polymorphonuclear leukocytes with few plasma cells [32]. Polymorphonuclear leucocytes with osteoclasts and necrosis are observed during early stages. Subsequently, lymphocytes and plasma cells predominate followed by fibrosis and signs of reactive new bone forming around the inflammation [18]. In more detailed studies there was a predominance of CD3+, CD45RO+ T cells, which were mainly CD4+ and CD8+ with low CD20+ B cell infiltration and abundance of CD68+ monocytes or macrophages [11]. In the course of an inflammatory process reparative changes of the osseous tissue such marrow fibrosis, trabecular osteoid apposition and periosteal hyperostosis are prominent. Granulocytes are more commonly present in CRMO than in unifocal non-recurrent or in multifocal non-relapsing lesions. Hyperostosis is more commonly found in CRMO than in unifocal non-recurrent lesions.

\section{Spinal manifestations in chronic recurrent multifocal osteomyelitis}

In gene-related infantile type of CRMO the spinal involvement is observed in $60 \%$ of patients, who may have a permanent deformity of the spine, such as vertebral fusion, nonunion of the odontoid with the atlas (C1), axis (C2) subluxation, and vertebral collapse leading to gibbus deformity [33]. Non-gene-related CRMO may have a variable course. The reported locations range from the mid-cervical to the sacral spine [34]. Osteomyelitis which causes localized pain may be the cause of mild scoliosis without vertebral rotation prior to any destruction of vertebrae $[26,35]$. Vertebral involvement may lead to collapse with subsequent vertebra plana or other deformities [19, 23, 29]. The typical osseous manifestations are: premature epiphyseal fusion, long bone deformity, growth arrest and progressive kyphosis [28, 35].

\section{Radiological presentation of chronic recurrent multifocal osteomyelitis in the spine}

The characteristic image includes bone oedema, lytic areas, periosteal reaction and soft tissue reaction with the possibility of the absence of periosteal reaction [6, 18]. Vertebral lesions often appear more sclerotic or osteolytic lesions with surrounding sclerosis may be present $[6,29]$. The radiological feature differentiating sterile osteomyelitis from spondyloarthropathies is spondylo-
A

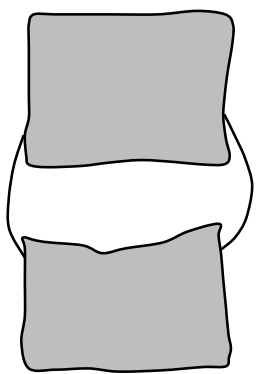

B

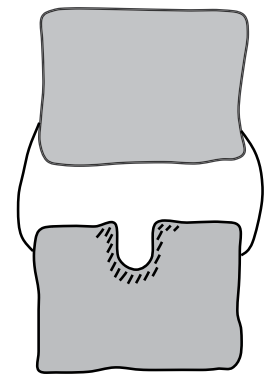

C

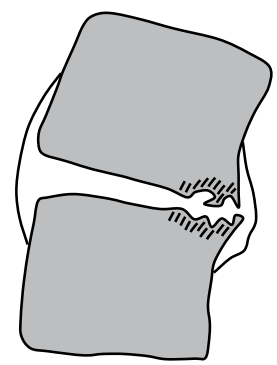

D

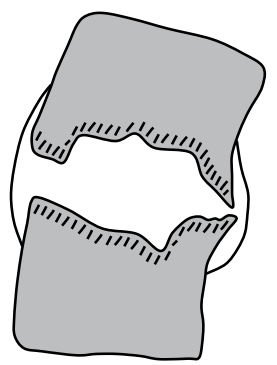

E

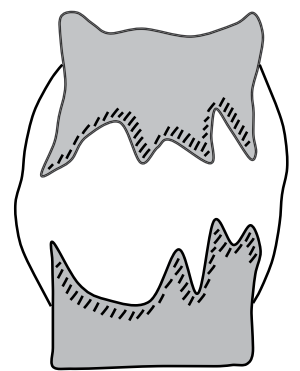

Fig. 1. Schematic types of radiological changes observed in the vertebral body morphology with the chronic non-bacterial osteomyelitis (CNO). 

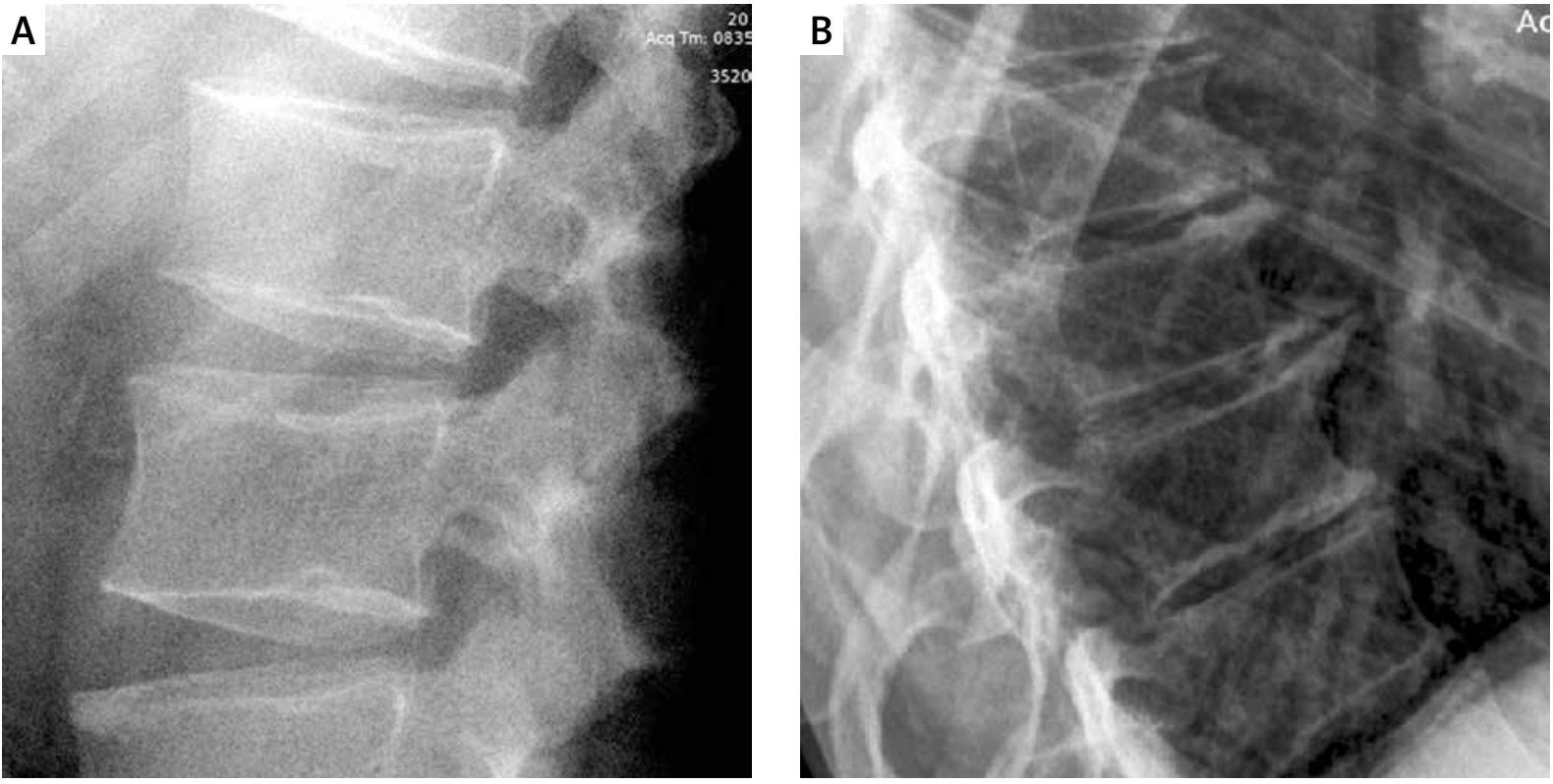

Fig. 2. Radiological presentation of mild chronic non-bacterial osteomyelitis (CNO) changes of vertebral bodies. Multi-level affections with end-plate remodeling, sclerotisation and anterior vertebral body height reduction (A). Characteristic vertebral body remodeling with bone hypertrophy and osteophyte formation (B).

discitis developing as a secondary destruction following spondylitis, although in some cases it cannot be clearly defined. The types of radiological changes observed in vertebral morphology with CNO are presented in Figures 1 and 2.

Vertebral deformity due to compression and total collapse (vertebra plana) are incidentally described in CRMO group $[35,36]$. Significant deformation may ultimately lead to neurological symptoms including spinal cord compression with paralysis [32, 37]. Computed tomography (CT) has a limited role in the diagnosis of CRMO [38]. CT findings correspond to those described under radiographic assessment, with the advantage of detecting subtle bone lesions, especially in radiologically obscure sites like the sternum, spine, and pelvis. Sclerosis and periosteal reaction are also better determined with CT examination. Magnetic resonance imaging (MRI) is the most sensitive imaging modality in short tau inversion recovery (STIR) post-gadolinium T1-weighted sequence showing a pathological signal [5, 39]. MRI scans also silent asymptomatic lesions to be revealed [6].

\section{Chronic recurrent multifocal osteomyelitis treatment in the spine}

Treatment options include non-steroidal anti-inflammatory drugs (NSAIDs), steroids and bisphosphonates [18]. Biological treatment was studied in resistant cases [40]. Operative treatment is rarely indicated. It was described in a case report with decompression and realignment with bone graft and anterior plate in the up- per thoracic spine [32]. In a larger study of 30 patients non-invasive treatment was necessary and good results were observed with naproxen and glucocorticoids regime [14]. In several cases good results were obtained after bisphosphonate administration with the complete resolution of lesions in MRI studies [39, 41].

\section{SAPHO syndrome}

SAPHO syndrome occurs in middle-aged adults $[1,5]$. Nevertheless, some authors include CRMO as a part of $\mathrm{SAPHO}$ syndrome. Therefore, age span may be respectively wider. The key symptoms are: synovitis (with affected joint swelling and limitation of movement), acne, pustulosis (most often the PPP form), hyperostosis and osteitis. There is a number of diagnostic criteria for SAPHO syndrome, but it is worth to notice that criteria made by Kahn (modified in 2003) seem to be the most precise (Table I) [42].

Not all the symptoms are concomitant, so not all are required for making a diagnosis, although linking PPP with osteitis is considered a strong diagnostic criterion [42]. The most commonly affected site in the skeleton is the anterior chest (with frequently observed sternocostoclavicular hyperostosis), followed by the spine [6]. According to some authors, the spine is one of the most commonly affected sites with the incidence ranging from $32 \%$ to $52 \%[7,10,43]$. The interval separating the onset of the symptoms and the diagnosis of SAPHO syndrome may be similar to the one in CRMO, i.e. approximately 6 or 9 years $[1,10]$. It is the most common in cas- 
Table I. Diagnostic criteria for SAPHO syndrome diagnosis proposed by Kahn [42], modified in 2003 [53] (from Kahn; American College of Rheumatology $67^{\text {th }}$ Annual Scientific Meeting, October 2003)

\begin{tabular}{|c|c|}
\hline Inclusion criteria & Exclusion criteria \\
\hline $\begin{array}{l}\text { - Bone-joint involvement associated with PPP and psoriasis } \\
\text { vulgaris } \\
\text { - Bone-joint involvement associated with severe acne } \\
\text { - Isolated sterilea hyperostosis/osteitis (adults) Chronic recur- } \\
\text { rent multifocal osteomyelitis (children) } \\
\text { - Bone-joint involvement associated with chronic bowel dis- } \\
\text { eases }\end{array}$ & $\begin{array}{l}\text { - Infectious osteitis } \\
\text { - Tumoral conditions of the bone } \\
\text { - Noninflammatory condensing lesions of the bone }\end{array}$ \\
\hline
\end{tabular}

es where a cutaneous lesion appears a long time after the onset of an osteoarticular lesion, or if skin problems do not appear at all [2]. In extreme cases this period may be 20 -year long [44]. Generally, in $50 \%$ of cases the vertebral and thoracic lesions preceded skin symptoms [7]. The clinical course of SAPHO syndrome often fluctuates between periods of recovery and deterioration [42].

Differential diagnosis includes infectious diseases, primary and metastatic tumours, diffuse idiopathic skeletal hyperostosis or retinoid therapy causing osseous deformity, osteosarcoma, Paget's disease, and POEMS (polyneuropathy, organomegaly, endocrinopathy, monoclonal gammopathy, and skin changes) syndrome [1]. In some cases the Propionibacterium acne was cultured with a possible indication on an infectious background of the syndrome [45-47]. However, recently the presence of the bacteria has been confirmed in other changes related to the degenerative process of the intervertebral disc $[48,49]$. Linking the species with either condition is controversial and its presence in both groups should be considered a co-occurrence rather than etiological factor [50].

\section{Natural history of SAPHO syndrome}

Spinal lesions in SAPHO syndrome are generally connected with a good prognosis. In some cases the vertebral destruction may cause a deformity and neurological problems, ultimately including paralysis resulting in a surgical intervention [1].

\section{Histopathological examination in SAPHO syndrome}

The histopathological examination of bone biopsy shows: specific granulation tissue with significant infiltration of, mostly lymphocytic, small round cells consistent with osteomyelitis, and plasmacytes, small round cell infiltrations, derangement of bony trabeculae and moderately increased osteoid, replacement of bone by fibrous tissue picture of chronic osteomyelitis with cellular infiltrate composed of lymphocytes and poly- morphonuclear leucocytes, areas of microabscesses, no specific lesions or inflammatory cells, especially in the disk space [7, 12]. In operative field spinal changes are fragile, resembling rheumatoid arthritis but including some sclerotic areas.

\section{Spinal presentation in SAPHO syndrome}

The spinal symptoms in SAPHO syndrome may be of great variety. The most common symptoms include low back pain and deep palpation tenderness, numbness and radial pain and sciatica, Lasègue's sign [2, 7, 12]. Sacroiliitis is observed in $13-52 \%$ of cases [7]. There are rare cases of quadriplegia due to the destruction of vertebrae followed by spinal cord compression. In some cases no neurological symptoms are observed [7]. Spinal lesions in SAPHO syndrome are common with variable occurrence in different studies: $15.7 \%, 32 \%$ and 52\% [7, $9,43]$. The mean age of the patients ranges from 47 to 51 years [12, 42]. Observations of few cases of spinal manifestations showed the prevalence of females among patients: $66.6 \%$ and $92 \%[1,12]$. The interval between the initial symptoms and diagnosis is 6.4 years [1]. There is usually a uniform distribution of occurrence in the whole spine with the minority of patients with one region of the spine affected [7]. In a group of eight patients three patients had cervical spine involvement, two patients - cervical and thoracic spine involvement, one patient - cervical, thoracic and lumbar spine involvement, three patients - cervical and lumbar spine involvement and four patients - thoracic and lumbar involvement [1].

\section{Radiological presentation of the SAPHO syndrome in the spine}

The radiological presentation of spondylodiscitis in SAPHO syndrome includes a spectrum of changes from mild fragmentary end-plate destruction to whole vertebra destruction and remodeling (Fig. 3). Vertebral lesions may begin in the end-plates as destructive, erosive and sclerotic remodeling, but it may also start at the vertebral body, with disc space preservation, or spread to the 
disc with its narrowing $[7,51]$. It was hypothesized, that enthesitis leads to osteolysis, erosion, synovitis, hyperostosis, with synostosis and ankylosis [43]. Another radiological analysis pointed out vertebral corner erosion as the primary change in MRI examination [52]. Multiple sites of spondylodiscitis (Fig. 4) in the same patient facilitate distinguishing the disease from infectious spondylodiscitis [7].

Severe destruction may lead to a kyphotic deformity with neurological complications. Slowly progressive non-marginal syndesmophytes at multi-spinal levels or a non-specific osteophyte pattern, which is characteristic for psoriatic spondylitis are observed in SAPHO syndrome [1]. Non-marginal syndesmophytes do not develop from the vertebral angle but from the middle of one vertebral body and extend to the same area of the adjacent vertebra [53]. A strong tendency towards ossification with the ultimate formation of ankylosing spinal hyperostosis is observed in advanced stages.

In some cases the presentation may resemble diffuse idiopathic skeletal hyperostosis (DISH) syndrome [12]. Diffuse idiopathic skeletal hyperostosis commonly causes the ossification of the posterior longitudinal ligament and ligamentum flavum, with common secondary neurological symptoms, without the evidence of inflammatory changes observed in MRI examination. According to Courtois et al. [54] the vertebral end-plate changes

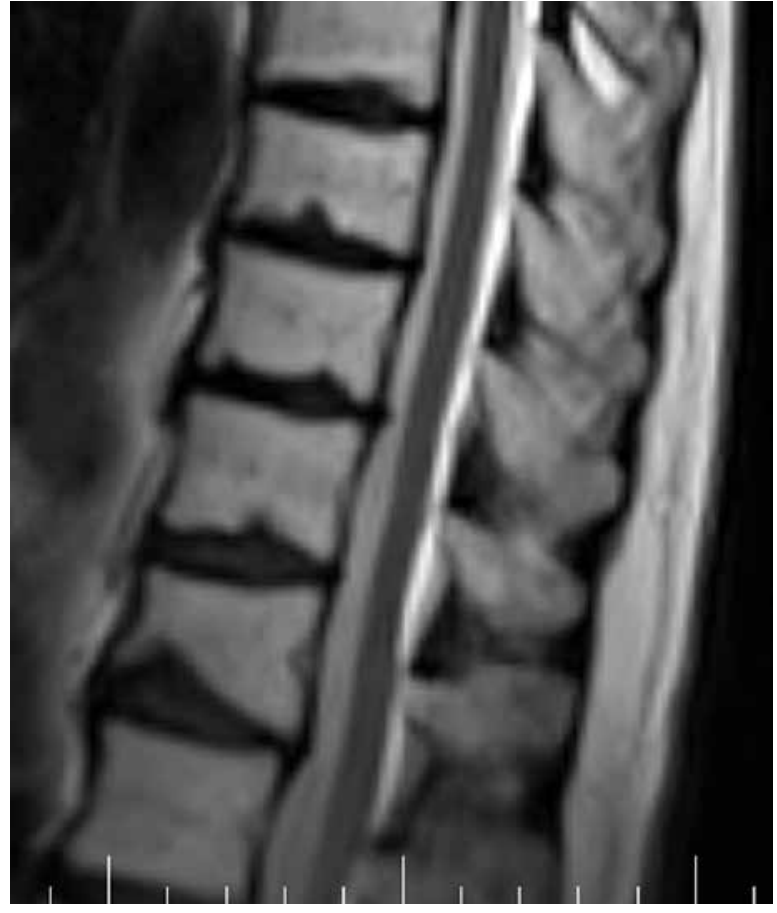

Fig. 3. T2 weighted magnetic resonance images shows typical persistent changes of chronic non-bacterial osteomyelitis (CNO) in thoracic spine. Normal signal intensity of the vertebral bodies with multi-level remodeling of the end-plates.
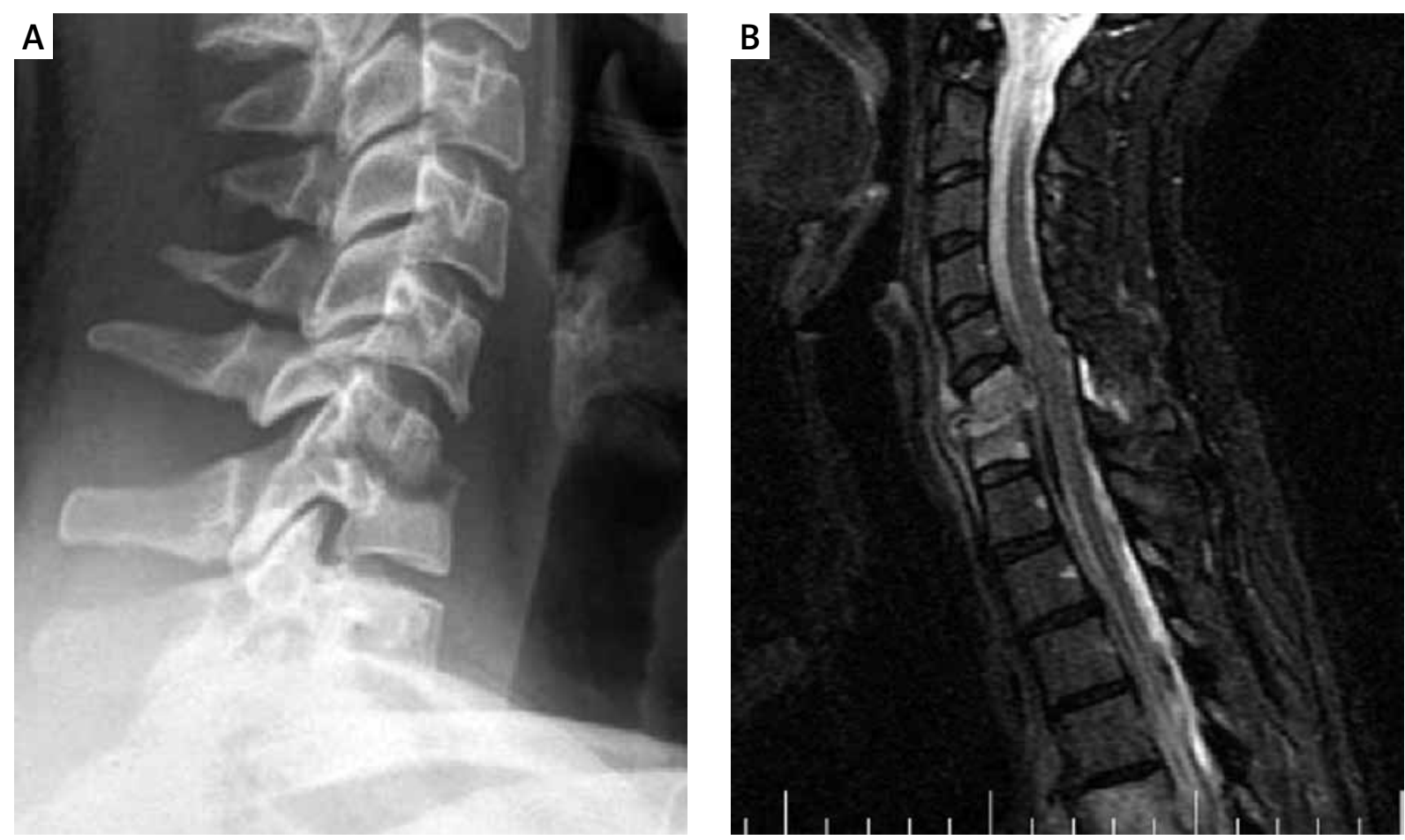

Fig. 4. Advanced spondylodiscitis at C6/C7 motion segment with end-plate destruction and local kyphosis (A). T2 weighted magnetic resonance images shows circumstantially, additionally to advanced destruction, abnormal signal in vertebrae suggesting chronic non-bacterial osteomyelitis (CNO) (B). 
are similar to those observed in CRMO. CT examination does not always show lesions typical for osteomyelitis and may be interpreted as degenerative [2]. It usually shows erosive lesions with no abscess [7]. In MRI examination abnormal signal intensity was most frequently noted in the vertebral end-plate with low-intensity in $\mathrm{T} 1$ and high-intensity in T2 images, enhanced after an injection of gadolinium with the sole involvement of vertebral bodies or together with the disk, depending on the extent of the lesion [7]. Non-consecutive, multi-level (more than three) spinal lesions were observed in 38\% of cases [1]. Abscesses and epiduritis, typical for infectious spondylodiscitis, were not observed on MRI scans. The follow up MRI usually showed no complications such as recurrences, or new location of spondylodiscitis. Studies usually show remodeling sequelae of spondylodiscitis [7]. The first differential diagnosis in SAPHO syndrome, based on MRI scans, may be one of multi-metastatic lesion due to the age of patients [55].

\section{SAPHO syndrome treatment}

Antimicrobial therapy is usually ineffective $[42,56]$. NSAID treatment is sufficient in most cases $[7,57,58]$. Systemic corticosteroids are used in unresponsive patients. According to the literature, successful treatment with sulphasalazine, colchicine, methotrexate, minocycline and biological treatment with anti-TNF therapy and even anti-interleukin-1 was conducted. Biological drugs have been used in selected cases [59-61]. Ultimately, surgical treatment is necessary [12]. The operative treatment rate is from 0 to $44 \%$ [7, 12]. Decisions for operative treatment were based mainly on neurological symptomatology with the goal to restore stability [12]. The authors stated: "When the vertebral lesion is acute and rapidly progressive, surgical intervention is recommended to diminish the cutaneous presentation and regain spinal stability" and "surgery is indicated only if the lesion shows progressive osteomyelitic changes associated with significant cutaneous and spinal symptoms" [12]. Some interventions described were performed for the potential decompression and drainage of suppurative spondylodiscitis with no confirmation of typical osteomyelitic changes at the site [2]. The results of the operative treatment are positive with good incorporation of autologous bone graft reinforced with metal implants.

\section{Summary}

The above literature review of both CRMO and SAPHO syndrome shows similar clinical presentations, especially as regards CNO in the spine. The similarities includes the predominance of female patients, and the clinical and histological presentation. Spinal involvement and "penetrance" in both age groups seems to be comparable with more extensive neurological symptomatology presented in the literature in the SAPHO syndrome group. This, in turn, may be ground for a higher number of surgical interventions in SAPHO syndrome patients. Despite the lack of reliable epidemiological studies, the number of CNO cases in the spine presented with the background of CRMO and SAPHO syndrome seems to be a noticeable medical problem. The concern is mainly about the diagnostic stage. An erroneous suspicion of the microbial etiology of the lesion may result in unnecessary treatment not influencing the course of the disease. Therefore, radiologists and clinicians need to be aware of its clinical and imaging presentation to avoid morbidity associated with improper treatment.

The authors declare no conflict of interest.

\section{References}

1. Takigawa T, Tanaka M, Nakanishi K, et al. SAPHO syndrome associated spondylitis. Eur Spine J 2008; 17: 1391-1397.

2. Kotilainen P, Gullichsen RE, Saario R, et al. Aseptic spondylitis as the initial manifestation of the SAPHO syndrome. Eur Spine J 1997; 6: 327-329.

3. Giedion A, Holthusen W, Masel LF, Vischer D. Subacute and chronic "symmetrical" osteomyelitis. Ann Radiol (Paris) 1972; 15: 329-342.

4. Sasaki T. A case of osteomyelitis of the bilateral clavicles associated with pustulosis palmaris et plantaris. Rinsho Seikeigeka 1967; 2: 333-337.

5. Ferguson PJ, Sandu M. Current understanding of the pathogenesis and management of chronic recurrent multifocal osteomyelitis. Curr Rheumatol Rep 2012; 14: 130-141.

6. Hofmann SR, Roesen-Wolff A, Hahn G, Hedrich CM. Update: Cytokine Dysregulation in Chronic Nonbacterial Osteomyelitis (CNO). Int J Rheumatol 2012; 2012: 310206.

7. Toussirot E, Dupond JL, Wendling D. Spondylodiscitis in SAPHO syndrome. A series of eight cases. Ann Rheum Dis 1997; 56: 52-58.

8. Zimmermann-Górska I, Puszczewicz M, Pietrzak-Kaczmarek H, Kołczewska A. Osteitis as a symptom of SAPHO syndrome. Pol Arch Med Wewn 2003; 110: 755-758.

9. Chamot AM, Benhamou CL, Kahn MF, et al. Acne-pustulosis-hyperostosis-osteitis syndrome. Results of a national survey. 85 cases. Rev Rhum Mal Osteoartic 1987; 54: 187-196.

10. Hayem G, Bouchaud-Chabot A, Benali K, et al. SAPHO syndrome: a long-term follow-up study of 120 cases. Semin Arthritis Rheum 1999; 29: 159-171.

11. Girschick HJ, Mornet E, Beer M, et al. Chronic multifocal non-bacterial osteomyelitis in hypophosphatasia mimicking malignancy. BMC Pediatr 2007; $7: 3$.

12. Baba H, Uchida K, Wada M, et al. Vertebral lesions associated with palmoplantar pustulosis. Int Orthop 1997; 21: 19-24. 
13. Silva PC, Oliveira EF, Goldenzon AV, et al. Challenges in diagnosis and treatment of a case of SAPHO syndrome in. Ann Bras Dermatol 2011; 86 (4 Suppl 1): S46-49.

14. Girschick HJ, Raab P, Surbaum S, et al. Chronic non-bacterial osteomyelitis in children. Ann Rheum Dis 2005; 64: 279-285.

15. Kahn MF, Chamot AM. SAPHO Syndrome. Rheum Dis Clin N Am 1992; 18: 225-246.

16. Simm PJ, Allen RC, Zacharin MR. Bisphosphonate treatment in chronic recurrent multifocal osteomyelitis. J Pediatr 2008, 152: 571-575.

17. Bjorksten B, Boquist L. Histopathological aspects of chronic recurrent multifocal osteomyelitis. J Bone Joint Surg 1980; 62 376-380.

18. Roderick MR, Ramanan AV. Chronic recurrent multifocal osteomyelitis. Adv Exp Med Biol 2013; 764: 99-107.

19. Huber AM, Lam P-Y, Duffy CM, et al. Chronic recurrent multifocal osteomyelitis: clinical outcomes after more than five years of follow-up. J Pediatr 2002; 141: 198-203.

20. Scully C, Hodgson T, Lachmann H. Auto-inflammatory syndromes and oral health. Oral Dis 2008; 14: 690-699.

21. Insalaco A, Bozzola E, Campana A, et al. Chronic recurrent multifocal osteomyelitis: 17 case reports and literature review. Pediatric Rheumatology Online Journal 2008; 6 (Suppl 1): P188. doi:10.1186/1546-0096-6-S1-P188.

22. El-Shanti HI, Ferguson PJ. Chronic recurrent multifocal osteo myelitis: a concise review and genetic update. Clin Orthop Relat Res 2007; 462: 11-19.

23. Schultz C, Holterhus PM, Seidel A, et al. Chronic recurrent multifocal osteomyelitis in children. Pediatr Infect Dis J 1999; 18: 1008-1013.

24. Quon JS, Dzus AK, Leswick DA. Case study: chronic recurrent multifocal osteomyelitis in the femoral diaphysis of a young female. Case Rep Radiol 2012; 2012: 515761.

25. Monsour PA, Dalton JB. Chronic recurrent multifocal osteomyelitis involving the mandible: case reports and review of the literature. Dentomaxillofac Radiol 2010; 39: 184-190.

26. Deogaonkar K, Ghandour A, Jones A, et al. Chronic recurrent multifocal osteomyelitis presenting as acute scoliosis: a case report and review of literature. Eur Spine J 2008; 17 Suppl 2: S248-252.

27. Haque SA, Shad A, Ozdemirli M, et al. A thirteen year old female with primary $\mathrm{T}$-cell rich B-cell lymphoma of bone masquerading as chronic recurrent multifocal osteomyelitis. Pediatr Rep 2009; 1: e3.

28. King SM, Laxer RM, Manson D, Gold R. Chronic recurrent multifocal osteomyelitis: a noninfectious inflammatory process. Pediatr Infect Dis J 1987; 6: 907-911.

29. Khanna G, Sato TS, Ferguson P. Imaging of chronic recurrent multifocal osteomyelitis. Radiographics 2009; 29: 1159-1177.

30. Tsitsami E, Dermentzoglou V, Moschovi M, Chrousos GP. Chronic recurrent multifocal osteomyelitis with concomitant features of juvenile idiopathic arthritis. Case Rep Rheumatol 2011; 2011: 210795.

31. Oberc A, Sułko J. Chronic recurrent multifocal osteomyelitis (CRMO) in children -single-centre clinical observations and experience. Ortop Traumatol Rehabil 2014; 16: 297-305.

32. Baulot E, Bouillien D, Giroux EA, Grammont PM. Chronic recurrent multifocal osteomyelitis causing spinal cord compression. Eur Spine J 1998; 7: 340-343.
33. Aksentijevich I, Masters SL, Ferguson PJ, et al. An autoinflammatory disease with deficiency of the interleukin-1-receptor antagonist. N Engl J Med 2009; 360: 2426-2437.

34. Schilling F, Fedlmeier M, Eckardt A, Kessler S. Vertebral manifestation of chronic recurrent multifocal osteomyelitis (CRMO). Rofo 2002; 174: 1236-1242.

35. Brown T, Wilkinson RH. Chronic recurrent multifocal osteomyelitis. Radiology 1988; 166: 493-496.

36. von Kalle T, Heim N, Hospach T, et al. Typical patterns of bone involvement in whole-body MRI of patients with chronic recurrent multifocal osteomyelitis (CRMO). Rofo 2013; 185: 655-661.

37. Gedouin JE, Violas P, Chapuis M, Bracq H. Stiff painful spine in an 11-year-old girl with SAPHO syndrome. Rev Chir Orthop Reparatrice Appar Mot 2001; 87: 830-834.

38. Buck FM, Treumann TC, Winiker H, Strobel K. Chronic recurrent multifocal osteomyelitis (CRMO) with symmetric involvement of both femora: X-ray, bone scintigram, and MR imaging findings in one case. J Magnetic Resonance Imaging 2007; 26: 422-426.

39. Miettunen PM, Wei X, Kaura D, et al. Dramatic pain relief and resolution of bone inflammation following pamidronate in 9 pediatric patients with persistent chronic recurrent multifocal osteomyelitis (CRMO). Pediatr Rheumatol Online J 2009; 7: 2. doi: 10.1186/1546-0096-7-2.

40. Marangoni RG, Halpern AS. Chronic recurrent multifocal osteomyelitis primarily affecting the spine treated with anti-TNF therapy. Spine (Phila Pa 1976) 2010; 35: E253-256.

41. Gleeson H, Wiltshire E, Briody J, et al. Childhood chronic recurrent multifocal osteomyelitis: pamidronate therapy decreases pain and improves vertebral shape. J Rheumatol 2008; 35 : 707-712.

42. Kahn MF, Khan MA. The SAPHO syndrome. Baillieres Clin Rheum 1994; 8: 333-362.

43. Maugars Y, Berthelot JM, Ducloux JM, Prost A. SAPHO syndrome: a follow up study of 19 cases with special emphasis on enthesis involvement. J Rheumatol 1995; 22: 2135-2141.

44. Kahn MF, Bouvier M, Palazzo E, et al. Sternoclavicular pustulotic osteitis (SAPHO). 20-year interval between skin and bone lesions. J Rheumatol 1991; 18: 1104-1108.

45. Edlund E, Johnsson U, Lidgren L, et al. Palmoplantar pustulosis and sternocostoclavicular arthro-osteitis. Ann Rheum Dis 1988; 47: 809-815.

46. Gerster JC, Lagier R, Livio JJ. Propionibacterium acnes in a spondylitis with palmoplantar pustulosis. Ann Rheum Dis 1990; 49: 337-338.

47. Kotilainen P, Merilahti-Palo R, Lehtonen OP, et al. Propionibacterium acnes isolated from sternal osteitis in a patient with SAPHO syndrome. J Rheumatol 1996; 23: 1302-1304.

48. Albert HB, Sorensen JS, Christensen BS, Manniche C. Antibiotic treatment in patients with chronic low back pain and vertebral bone edema (Modic type 1 changes): a double-blind randomized clinical controlled trial of efficacy. Eur Spine J 2013; 22: 697-707.

49. Aebi M. Is low back pain after disc herniation with Modic Type 1 changes a low-grade infection? Eur Spine J 2013; 22: 689.

50. Birkenmaier C. Should we start treating chronic low back pain with antibiotics rather than with pain medications? Korean J Pain 2013; 26: 327-335. 
51. Earwaker JW, Cotten A. SAPHO: syndrome or concept? Imaging findings. Skeletal Radiol 2003; 32: 311-327.

52. Laredo JD, Vuillemin-Bodaghi V, Boutry N, et al. SAPHO Syndrome: MR Appearance of Vertebral Involvement. Radiology 2007; 242: 825-831.

53. Khan MA. Clinical features of ankylosing spondylitis. In: Rheumatology. Hochberg MC, Silman AJ, Smolen JS, et al. (eds.) Mosby, St. Louis 2003: 1161-1181.

54. Courtois C, Fallet GH, Vischer TL, Wettstein P. Erosive spondylopathy. Ann Rheum Dis 1980; 39: 462-468.

55. Mann B, Shaerf DA, Sheeraz A, et al. SAPHO syndrome presenting as widespread bony metastatic disease of unknown origin. Rheumatol Int 2012; 32: 505-507.

56. Benhamou CL, Chamot AM, Kahn MF. Synovitis-acne-pustulosis hyperostosis-osteomyelitis syndrome (Sapho). A new syndrome among the spondyloarthropathies? Clin Exp Rheumatol 1988; 6: 109-112.

57. Kaiser D, Bolt I, Hofer M, et al. Chronic nonbacterial osteomyelitis in children: a retrospective multicenter study. Pediatr Rheumatol Online J 2015; 13: 25. doi: 10.1186/s12969-0150023-y.

58. Heldmann F, Kiltz U, Baraliakos X, Braun J. SAPHO syndrome. Z Rheumatol 2014; 73: 729-741

59. Takizawa Y, Murota A, Setoguchi K, Suzuki Y. Severe inflammation associated with synovitis, acne, pustulosis, hyperostosis, osteitis (SAPHO) syndrome was markedly ameliorated by single use of minocycline. Mod Rheumatol 2014; 24: 1015-1018.

60. Hampton SL, Youssef H. Successful treatment of resistant SAPHO syndrome with anti-TNF therapy. BMJ Case Rep 2013; 2013. pii: bcr2012007161. doi: 10.1136/bcr-2012-007161.

61. Maślińska M. Zespół SAPHO - opis przypadku. Reumatologia 2005; 43: 222-226. 